\title{
LA PARADOJA DE LA EDUCACIÓN Y LA POLÍTICA PÚBLICA: LIMITACIONES EN LA POLÍTICA PÚBLICA PARA GENERAR UNA EDUCACIÓN INCLUSIVA Y LA NECESIDAD DE LA EDUCACIÓN PARA LA INCLUSIÓN SOCIAL
}

(The paradox of education and public policy: limitations on public policy to create an inclusive education and the educational need for

social inclusion)

Iván Rodrigo Baquero Micán'

Docente - Investigador

Corporación Educativa Indoamericana ivanbaquero33@gmail.com

Resumen

Abordar el tema de inclusión social en el contexto actual de nuestro país, en el cual se está llevando a cabo el proceso de paz, resulta complejo y a la vez necesario; complejo debido a varios factores entre los cuales se pueden señalar la polarización, la falta de identidad y fines comunes, la cultura de la indiferencia y el descarte, el individualismo, el facilismo y los fundamentalismos ideológicos; necesario porque si se analiza en profundidad la historia del conflicto en nuestro país ha estado enmarcado en las condiciones de injusticia, desigualdad, marginalidad y falta de políticas que permitan un auténtico desarrollo sostenible e incluyente y si se quiere alcanzar la paz, no bastará con la firma de un documento sino con un cambio real en las políticas públicas y en la mentalidad de cada colombiano como condición de posibilidad de la misma.

Palabras clave: política pública, inclusión social, proceso de paz, desigualdad, desarrollo sostenible.

Abstract

Addressing the issue of social inclusion in the current context of our country, which is holding the peace process is complex and at the same time necessary; it is complex due to several factors which may point at polarization, lack of identity and common purposes, culture of indifference and discarding, individualism, the easiness and ideological fundamentalism; necessary because if we analyze in depth the history of the conflict in our country has been framed in terms of injustice, inequality, marginalization and lack of policies to real sustainable and inclusive development and if we want to make peace, it will not be enough to sign a document, but a real change in public policy and in the minds of every Colombian as a condition for it.

Keywords: public policy, social inclusion, peace process inequality, sustainable development. 


\section{INTRODUCCIÓN}

A continuación se presenta un pequeño análisis referente a las condiciones contextuales quese erigieron como causas del conflicto. Para que una sociedad sea catalogada como justa, no se debe comprender bajo el concepto de igualdad, ya que esta no siempre es garantía de justicia; esto se evidencia con claridad en la misma sociedad y, más específicamente, en un aula de clase: si se anunciara a los estudiantes que, para que haya una auténtica igualdad, como resultado de una evaluación a todos se les asignará la misma nota, de inmediato surgirán voces de protesta con absoluta razón ipor qué deben obtener la misma nota los que se han esforzado, los que han realizado sus trabajos y adquirido las competencias y los que no? De la misma manera ocurre en la sociedad con los modelos asistencialistas que, además, son un principio del Estado social de derecho en el cual se inscribe nuestro país; ipor qué razón una persona que no trabaja debe gozar gratuitamente de lo que los demás consiguen con mucho esfuerzo y sacrificio? ¿Cómo es posible que obtengan los mismos beneficios los que se esfuerzan y los que no? Incluso, algunos autores señalan que el orden jerárquico piramidal de la sociedad en los Estados de derecho se está invirtiendo, ya que la clase media está pasando a la base misma de la estructura. Por ello, una sociedad justa es aquella que garantiza la movilidad social, es decir, en la que las personas que se esfuerzan y trabajan por salir adelante, se proyectan y lo dan todo, respetando las normas, los parámetros comportamentales, la libertad y la dignidad humana, puedan ascender.

\section{LA PARADOJA DE LA EDUCACIÓN Y LA POLÍTICA PÚBLICA}

Al intentar comprender la relación entre educación y política pública surgen una serie de conflictos que se pueden evidenciar en la siguiente pregunta: icómo hablar de justicia si no todos parten del mismo punto y unos ya tienen una ventaja sobre los demás?; ante esto, el sociólogo Durkheim planteaba la necesidad de suspender la herencia; además de ello, se requeriría que los derechos fundamentales de las personas estuviesen garantizados como condición necesaria para el desarrollo y allí radicaría la intervención del Estado. Sin embargo, en Colombia existen otros problemas que dificultan esta tarea; desde el surgimiento de la nación se ha favorecido la acumulación de riquezas, la marginalidad y esto se ha convertido en parte de la misma identidad e idiosincrasia; pero, además de ello, y por diversos factores entre los cuales también se deben señalar los temas referentes a las recomendaciones del fondo
Monetario Internacional, el consenso de Washington, los tratado de libre comercio, la carga tributaria, la falta de infraestructura y la ausencia de incentivos adecuados para la generación de industria, la verdad es que el patrimonio de los colombianos se ha centrado en activos fijos y no en inversiones que generen empleo y que dinamicen realmente la economía; el crecimiento de la industria es cada vez menor y no es proporcional a los requerimientos de una economía sostenida. Los sectores económicos en los que participamos se limitan a la minería y la explotación de materia prima impidiendo el crecimiento del PIB y dificultando el desarrollo de la política pública. En un país en el que el ingreso per cápita de los colombianos es de $\$ 578422$,en el que solo un $34 \%$ de la población supera los dos salarios mínimos legales vigentes, en el que el desempleo alcanza al $9,6 \%$, el subempleo el $39,9 \%$ y la informalidad el $51,6 \%$, resulta necesario hablar de equidad y desarrollo pero, a la vez, es realmente inviable pensar en grandes posibilidades. A esa minoría de colombianos, que ganan más de dos salarios mínimos, hay que cargarlos con impuestos pues no hay más opción, tanto como a los que poseen un patrimonio superior a los mil millones de pesos, que en Colombia son tan solo el uno por ciento de la población. En ese orden de ideas, llegará el momento en que los impuestos se deban aplicar a capitales muy inferiores al monto actual.

Ante esta realidad, debemos recordar que el medio más idóneo para buscar soluciones a dicha problemática es la política pública la cual es definida por Gavilanes (2009) como:

(...) Un proceso integrador de decisiones, acciones, inacciones, acuerdos e instrumentos, adelantado por autoridades públicas con la participación eventual de los particulares, y encaminado a solucionar o prevenir una situación definida como problemática. La política pública hace parte de un ambiente determinado del cual se nutre y al cual pretende modificar o mantener (p. 156).

La política pública, entonces, tiene como objetivo generar normativas para la protección del bien común proponiendo solución a problemas públicos; pero las preguntas fundamentales que surgen son: ipara quién se hace la política pública?, ¿para quién debería hacerse?, ¿qué criterio se emplea para la elaboración de la política pública? En algunas ocasiones se considera que la política pública debe hacerse para solucionar las problemáticas de la población vulnerable y de las minorías, con lo cual se puede caer fácilmente en el asistencialismo. Por otro lado, otros consideran que las políticas públicas deben centrarse en el crecimiento y para ello se debe 
incentivar a los que más tienen para activar la economía y generar un efecto cascada que terminará beneficiando a los menos favorecidos con mayores posibilidades de empleo.

Sin embargo, lo más acertado sería adoptar una postura intermedia: favorecer a las personas vulnerables pero no con la promoción de un sistema asistencialista, sino brindándoles herramientas para que ingresen a los sectores productivos y competitivos mediante un sistema educativo incluyente y de calidad, ya que es la manera de conseguir una democratización económica que es, a su vez, la condición necesaria para una democratización política y social, pero, adicionalmente, garantizando la justicia, la seguridad, el acceso a las prestaciones de ley (salud y pensión) y, en general, la infraestructura necesaria para el desarrollo del país (Gavilanes, 2009).

Si el esfuerzo del Estado se centrara en los temas señalados, se calcula que con una inversión del 1,4\% más del PIB se podrían garantizar los mínimos en lo referente a la cobertura de educación; en la actualidad se invierte el 4,6\% del PIB según datos del Banco Mundial. Con una inversión del I,3 \% más del PIB se podría garantizar la asistencia básica; en la actualidad se encuentra en el 6,8 \% del PIB. Con un aumento del 3,5\% del porcentaje actual del PIB, que es del 0,5 $\%$, se podría garantizar el seguro pensional (Gavilanes, 2009). Esto equivale en realidad a menos del $18 \%$ del PIB; sin embargo, en la actualidad el 57,4 \% del PIB es empleado para gastos de funcionamiento del Estado, el 22,2 \% para pago de la deuda externa y el 20,3\% para inversión. De los anteriores datos se puede inferir que se requiere del $100 \%$ del presupuesto nacional destinado a la inversión para mejorar las condiciones de los colombianos, sin desconocer que existen otras inversiones que resultan también prioritarias como la infraestructura y los incentivos para la creación de industria. La única manera de lograr un desarrollo sostenible es disminuir considerablemente los gastos de funcionamiento del Estado y esto, lastimosamente, no se ha considerado realmente en las políticas estatales.

\section{LA EDUCACIÓN INCLUSIVA Y LA NECESIDAD \\ DE LA EDUCACIÓN PARA LA INCLUSIÓN SOCIAL}

Poder realizar una adecuada valoración de la educación, en relación con la desigualdad, implica dejarla de ver como un problema y un índice de exclusión, para asumirla como herramienta, siendo la condición necesaria reemplazar la comprensión de ella como gasto para entenderla como inversión, pues se requiere no solo garantizar la cobertura, sino la calidad, con un enfoque que no se reduzca a la generación de mano de obra capacitada y sumisa, sino a la creación de empresa, de industria, de modelos para el desarrollo sostenible, de innovación, investigación y tecnología; solo así la educación podrá transformarse en mecanismo, dejando de ser solo un indicador de inclusión social; pero, para "(...) que la educación ayude a la gente pobre a salir de la pobreza, antes hay que ayudar a que la educación salga de la pobreza en que se encuentra" (De Zubiría, 2009, Conclusiones, § 4).

Ahora bien, se debe abandonar la idea de que una educación inclusiva consiste en "enseñar a todos todo"; se trata de una falacia, ya que las situaciones contextuales, los intereses, los puntos de partida y las finalidades de la educación de las personas varían y, por ello, lo que para unos es adecuado y suficiente, para otros no lo es; ignorar esta consideración implicará que la calidad de la educación se afecte y se terminará culpando a los estudiantes cuando en realidad se trata de un problema en la concepción y planeación de la misma (Gavilanes, 2009).

Otro grave problema de las concepciones erróneas de inclusión radicaría en la homogeneización, dejando de lado la pluralidad de modelos pedagógicos que son más o menos adecuados a las personalidades e intereses de los estudiantes, lo cual puede terminar constituyéndose como el principal motivo de exclusión.

\section{CONCLUSIÓN}

Por lo expuesto anteriormente, para que la educación sea inclusiva y a la vez se erija como herramienta para la superación de la exclusión, se requiere de la redistribución (ampliar los beneficios de la calidad educativa a la población según sus particularidades), el reconocimiento de las características y necesidades de la población de los diversos modelos y sus aportes y limitaciones, y la inversión de recursos que garanticen la infraestructura, la cobertura y la calidad.

En la actualidad se ha señalado que una educación solo puede ser de calidad si garantiza la inclusión de toda la población; sin embargo, el querer alcanzar una cobertura significativa ha traído como consecuencia la disminución en la calidad objetiva de la misma, lo cual exige docentes especializados tanto en la fundamentación epistemológica como pedagógica, y la independencia y el apoyo para el cumplimiento de su labor \& 


\section{Referencias bibliográficas}

De Zubiría, J. (Octubre 2 de 2014). ¿Ser pobres condena a los estudiantes?La 2 Orillas [en línea]. Recuperado de http://www.las2orillas.co/ ser-pobres-condena-los-estudiantes/.

Gavilanes, R. V. (ler Semestre, 2009). Hacia una nueva definición del concepto “política pública”. Desafíos, (20) I49-187. Versión en línea disponible en http://www.ins.gov.co/investigacion/docs/Hacia\%20una\%20nueva\%20definici\%C3\%B3n\%20de\%20pol\%C3\%ADtica\%20 p\%C3\%BAblica.pdf 